\title{
Hopf-cyclic homology and cohomology with coefficients
}

\author{
Piotr M. Hajac ${ }^{a}$, Masoud Khalkhali ${ }^{b}$, Bahram Rangipour $^{b}$, \\ Yorck Sommerhäuser ${ }^{c}$
}

a Instytut Matematyczny, Polska Akademia Nauk, ul. Śniadeckich 8, Warszawa, 00-956 Poland, and Katedra Metod Matematycznych Fizyki, Uniwersytet Warszawski, ul. Hoża 74, Warszawa, 00-682 Poland; URL: http://www.fuw.edu.pl/ pmh

$b$ Department of Mathematics, University of Western Ontario, London ON, Canada; E-mail: masoud@uwo.ca, brangipo@uwo.ca

c Mathematisches Institut, Universität München, Theresienstr. 39, 80333 München, Germany; E-mail: sommerh@mathematik.uni-muenchen.de

\begin{abstract}
Following the idea of an invariant differential complex, we construct general-type cyclic modules that provide the common denominator of known cyclic theories. The cyclicity of these modules is governed by Hopfalgebraic structures. We prove that the existence of a cyclic operator forces a modification of the Yetter-Drinfeld compatibility condition leading to the concept of a stable anti-Yetter-Drinfeld module. This module plays the role of the space of coefficients in the thus obtained cyclic cohomology of module algebras and coalgebras, and the cyclic homology and cohomology of comodule algebras. Along the lines of Connes and Moscovici, we show that there is a pairing between the cyclic cohomology of a module coalgebra acting on a module algebra and closed 0-cocycles on the latter. The pairing takes values in the usual cyclic cohomology of the algebra. Similarly, we argue that there is an analogous pairing between closed 0-cocycles of a module coalgebra and the cyclic cohomology of a module algebra.
\end{abstract}

\section{Homologie et cohomologie Hopf-cycliques à coefficients}

Résumé. Suivant l'idée d'un complexe différentiel invariant, nous construisons des modules cycliques de type général qui fournissent un dénominateur commun aux théories cycliques connues. Le caractère cyclique de ces modules est gouverné par des structures Hopf-algébriques. Nous montrons que l'existence d'un opérateur cyclique oblige à une modification de la condition de compatibilité de Yetter-Drinfeld et mène au concept de module antiYetter-Drinfeld stable. Ce module joue le rôle d'espace de coefficients pour la cohomologie de modules algèbres et de modules cogèbres ainsi obtenue, ainsi que pour l'homologie et la cohomologie cycliques de comodules algèbres. Comme l'ont fait Connes et Moscovici pour leur cohomologie, nous montrons qu'il existe un appariement entre la cohomologie cyclique d'un module cogèbre agissant sur un module algèbre et les 0-cycles fermés sur ce dernier. Cet appariement prend ses valeurs dans la cohomologie cyclique usuelle de l'algèbre. De façon similaire, nous établissons un appariement analogue entre les 0-cycles fermés d'un module cogèbre et la cohomologie cyclique d'un module algèbre.

\section{Version française abrégée.}

Soit $H$ une algèbre de Hopf avec une antipode bijective, et soit $M$ à la fois un module et un comodule sur $H$. Nous disons que $M$ est un module anti-Yetter-Drinfeld s'il satisfait à l'une des conditions équivalentes (1.1)-(1.4). Nous disons que $M$ est stable s'il a la propriété action o coaction $=\mathrm{id}$. Le résultat principal de cet article est la construction de modules cycliques qui nous permettent de définir la cohomologie cyclique de $H$-modules cogèbres et de $H$-modules algèbres à coefficients dans des modules anti-Yetter-Drinfeld stables. De plus, si un $H$-module cogèbre $C$ agit sur un $H$-module algèbre $A$ au sens où nous avons une application linéaire $C \otimes A \rightarrow A$ satisfaisant $c(a b)=\left(c^{(1)} a\right)\left(c^{(2)} b\right), c 1=\varepsilon(c), h(c a)=(h c) a$, pour tous $h \in H, c \in C$, alors il existe un certain appariement entre ces cohomologies pour $C$ et $A$. 
Theorem 0.1 Soit $H$ une algèbre de Hopf avec une antipode bijective et soit $M$ un module antiYetter-Drinfeld sur $H$ stable. Alors les formules (2.1)-(2.4) (resp. (2.6)-(2.9)) définissent une structure de module cyclique sur $\left\{M \otimes_{H} C^{\otimes(n+1)}\right\}_{n \in \mathbb{N}}\left(\right.$ resp. $\left.\left\{\operatorname{Hom}^{H}\left(M \otimes A^{\otimes(n+1)}, k\right)\right\}_{n \in \mathbb{N}}\right)$ pour tout $H$-module cogèbre $C$ (resp. algèbre $A$ ). Si de plus $C$ agit sur $A$ et $\mathbb{Q} \subseteq k$, alors, pour tout $n \in \mathbb{N}$, les formules (2.10) et (2.11) définissent, respectivement, les homomorphismes suivants:

$$
H C_{H}^{n}(C, M) \otimes H C_{H}^{0}(A, M) \stackrel{\#_{n, 0}}{\longrightarrow} H C^{n}(A), \quad H C_{H}^{0}(C, M) \otimes H C_{H}^{n}(A, M) \stackrel{\#_{0, n}}{\longrightarrow} H C^{n}(A) .
$$

De façon similaire, pour tout comodule algèbre, les formules (3.1)-(3.4) et (3.5)-(3.8) déterminent respectivement notre construction d'une homologie et d'une cohomologie cycliques à coefficients dans un module anti-Yetter-Drinfeld stable.

\section{Introduction}

Ever since its invention, among main applications of cyclic cohomology was the computation of $K$ theoretical invariants. While enhancing the feasibility of such computations, Connes and Moscovici discovered a new type of cyclic cohomology, notably the cyclic cohomology of Hopf algebras [6]. Invariant cyclic homology introduced in [11] generalizes the Connes-Moscovici theory and its dual version [12]. It shows that passage from the cyclic homology of algebras to the cyclic cohomology of Hopf algebras is remarkably similar to passage from de Rham cohomology to cohomology of Lie algebras via invariant de Rham cohomology [4]. The idea of employing invariant complexes proved to be a key in resolving by significantly more effective means the technical challenge of showing that the $(n+1)$-power of the cyclic operator $\tau_{n}$ is identity [7, p.102], and allowed the introduction of higher-dimensional coefficients.

We continue this strategy herein. Our motivation is to obtain and understand computable invariants of $K$-theory. The aim of this paper is to provide general framework for cyclic theories whose cyclicity is based on Hopf-algebraic structures. We refer to such homology and cohomology as Hopf-cyclic. First we define and provide sources of examples of stable anti-Yetter-Drinfeld modules that play the role of coefficients for Hopf-cyclic theory. In particular, we claim that modular pairs in involution are precisely 1-dimensional stable anti-Yetter-Drinfeld modules. Then we construct cyclic module structures on invariant complexes for module coalgebras and module algebras, respectively. It turns out that the cyclic cohomology of Hopf algebras is a special case of the former, whereas both twisted [13] and usual cyclic cohomology are special cases of the latter. As a bonus of generality, we obtain now a very short proof of Connes-Moscovici key result [7, Theorem 1]. Furthermore, as $\delta$-invariant $\sigma$-traces can be viewed as closed 0 -cocycles on a module algebra, our pairing for Hopf-cyclic cohomology generalizes the Connes-Moscovici transfer map [7, Proposition 1] from the cyclic cohomology of Hopf algebras to ordinary cyclic cohomology. Finally, we end this article by deriving Hopf-cyclic homology and cohomology of comodule algebras. This extends the formalism for comodule algebras provided in [11].

Partly for the sake of simplicity, throughout the paper we assume that $H$ is a Hopf algebra with a bijective antipode. On one hand side, the bijectivity of the antipode is implied by the existence of a modular pair in involution, so that then it need not be assumed. On the other hand, some parts of arguments might work even if the antipode is not bijective. We avoid such discussions. The coproduct, counit and antipode of $H$ are denoted by $\Delta, \varepsilon$ and $S$, respectively. For the coproduct we use the notation $\Delta(h)=h^{(1)} \otimes h^{(2)}$, for a left coaction on $M$ we write ${ }_{M} \Delta(m)=m^{(-1)} \otimes m^{(0)}$, and for a right coaction $\Delta_{M}(m)=m^{(0)} \otimes m^{(1)}$. The summation symbol is suppressed everywhere. We assume all algebras to be associative, unital and over the same ground field $k$. The symbol $\mathcal{O}(X)$ stands for the algebra of polynomial functions on $X$. 


\section{Stable anti-Yetter-Drinfeld modules}

It turns out that, in order to incorporate coefficients into cyclic theory, we need to alter the concept of a Yetter-Drinfeld module by replacing the antipode by its inverse in the Yetter-Drinfeld compatibility condition between actions and coactions. We call the modules-comodules satisfying the thus modified Yetter-Drinfeld compatibility condition anti-Yetter-Drinfeld modules ${ }^{1}$. Just as Yetter-Drinfeld modules come in 4 different versions depending on the side of actions and coactions (see [3, p.181] for a general formulation), so do the anti-Yetter-Drinfeld modules. All versions are completely equivalent and can be derived from one another by replacing a Hopf algebra $H$ by $H^{c o p}$, $H^{o p}$, or $H^{o p, c o p}$, respectively. Explicitly, we have the following:

Definition 1.1 Let $H$ be a Hopf algebra with a bijective antipode $S$, and $M$ a module and comodule over $H$. We call $M$ an anti-Yetter-Drinfeld module iff the action and coaction are compatible in the following sense:

$$
\begin{aligned}
& { }_{M} \Delta(h m)=h^{(1)} m^{(-1)} S^{-1}\left(h^{(3)}\right) \otimes h^{(2)} m^{(0)} \quad \text { if } M \text { is a left module and a left comodule, } \\
& \Delta_{M}(h m)=h^{(2)} m^{(0)} \otimes h^{(3)} m^{(1)} S\left(h^{(1)}\right) \quad \text { if } M \text { is a left module and a right comodule, } \\
& { }_{M} \Delta(m h)=S\left(h^{(3)}\right) m^{(-1)} h^{(1)} \otimes m^{(0)} h^{(2)} \quad \text { if } M \text { is a right module and a left comodule, } \\
& \Delta_{M}(m h)=m^{(0)} h^{(2)} \otimes S^{-1}\left(h^{(1)}\right) m^{(1)} h^{(3)} \quad \text { if } M \text { is a right module and a right comodule. }
\end{aligned}
$$

To make cyclic theory work, we also need to assume that the action splits coaction, i.e., for all $m \in M, m^{(-1)} m^{(0)}=m, m^{(1)} m^{(0)}=m, m^{(0)} m^{(-1)}=m, m^{(0)} m^{(1)}=m$, for the left-left, left-right, right-left, and right-right version respectively. We call modules satisfying this condition stable. Let us emphasize that it is the anti-Yetter-Drinfeld condition rather than the Yetter-Drinfeld condition that makes the homomorphism action $\circ$ coaction $H$-linear and $H$-colinear. Therefore the stability condition action $\circ$ coaction $=$ id suits the former and not the latter. The first class of examples of stable anti-Yetter-Drinfeld modules is provided by modular pairs in involution [8, p.8].

Lemma 1.2 Let the ground field $k$ be a right module over $H$ via a character $\delta$ and a left comodule over $H$ via a group-like $\sigma$. Then $k={ }^{\sigma} k_{\delta}$ is a stable anti-Yetter-Drinfeld module if and only if $(\delta, \sigma)$ is a modular pair in involution.

This combined with the following lemma guarantees a rich source of examples of anti-YetterDrinfeld modules.

Lemma 1.3 Let $N$ be a Yetter-Drinfeld module and $M$ an anti-Yetter-Drinfeld module. Then $N \otimes M$ is an anti-Yetter-Drinfeld module via $h(n \otimes m)=h^{(1)} n \otimes h^{(2)} m, N \otimes M \Delta(n \otimes m)=$ $n^{(-1)} m^{(-1)} \otimes n^{(0)} \otimes m^{(0)}$, for the left-left case, and via $h(n \otimes m)=h^{(2)} n \otimes h^{(1)} m, \Delta_{N \otimes M}(n \otimes m)=$ $n^{(0)} \otimes m^{(0)} \otimes n^{(1)} m^{(1)}$, for the left-right case. Similarly, $M \otimes N$ is an anti-Yetter-Drinfeld module via $(m \otimes n) h=m h^{(2)} \otimes n h^{(1)}, M \otimes N \Delta(n \otimes m)=m^{(-1)} n^{(-1)} \otimes m^{(0)} \otimes n^{(0)}$, for the right-left case, and via $(m \otimes n) h=m h^{(1)} \otimes n h^{(2)}, \Delta_{M \otimes N}(m \otimes n)=m^{(0)} \otimes n^{(0)} \otimes m^{(1)} n^{(1)}$, for the left-right case.

An intermediate step between modular pairs in involution and stable anti-Yetter-Drinfeld modules is given by matched and comatched pairs of [11]. Whenever the antipode is equal to its inverse, the difference between the Yetter-Drinfeld and anti-Yetter-Drinfeld conditions disappears. For a group ring Hopf algebra $k G$, a left $H$-comodule is simply a $G$-graded vector space $M=\bigoplus_{g \in G} M_{g}$, where the coaction is defined by $M_{g} \ni m \mapsto g \otimes m$. An action of $G$ on $M$ defines an (anti-) Yetter-Drinfeld module if and only if for all $g, h \in G$ and $m \in M_{g}$ we have $h m \in M_{h g h^{-1}}$. The stability condition means simply that $g m=m$ for all $g \in G, m \in M_{g}$. A very concrete

\footnotetext{
${ }^{1}$ This concept was devised independently by Ch. Voigt and, also independently, by P. Jara and D. Stefan.
} 
classical example of a stable (anti-)Yetter-Drinfeld module is provided by the Hopf fibration. Then $H=\mathcal{O}(S U(2))$ and $M=\mathcal{O}\left(S^{2}\right)$. Since $S^{2} \cong S U(2) / U(1)$, we have a natural left action of $S U(2)$ on $S^{2}$. Its pull-back makes $M$ a left $H$-comodule. On the other hand, one can view $S^{2}$ as the set of all traceless matrices of $S U(2)$. The pull-back of this embedding $j: S^{2} \hookrightarrow S U(2)$ together with the multiplication in $\mathcal{O}\left(S^{2}\right)$ defines a left action of $H$ on $M$. It turn out that the equivariance property $j(g x)=g j(x) g^{-1}$ guarantees the anti-Yetter-Drinfeld condition, and this combined with the injectivity of $j$ ensures the stability of $M$. This stability mechanism can be generalized in the following way.

Lemma 1.4 Let $M$ be an algebra and a left $H$-comodule. Assume that $\pi: H \rightarrow M$ is an epimorphism of algebras and the action $h m=\pi(h) m$ makes $M$ an anti-Yetter-Drinfeld module. Assume also that $\pi\left(1^{(-1)}\right) 1^{(0)}=1$. Then $M$ is a stable module.

Another source of examples is provided by Hopf-Galois theory. These examples are purely quantum in the sense that the employed actions are automatically trivial for commutative algebras. To fix the notation and terminology, recall that an algebra and an $H$-comodule is called a comodule algebra if the coaction is an algebra homomorphism. An $H$-extension $B:=\left\{p \in P \mid \Delta_{P}(p)=\right.$ $p \otimes 1\} \subseteq P$ is called Hopf-Galois iff the canonical map can $: P \otimes_{B} P \rightarrow P \otimes H, \operatorname{can}\left(p \otimes p^{\prime}\right)=p \Delta\left(p^{\prime}\right)$, is bijective. The bijectivity assumption allows us to define the translation map $T: H \rightarrow P \otimes_{B} P$, $T(h):=\operatorname{can}^{-1}(1 \otimes h)=: h^{[1]} \otimes_{B} h^{[2]}$ (summation suppressed). It can be shown that when everything is over a field (our standing assumption), the centralizer $Z_{B}(P):=\{p \in P \mid b p=p b, \forall b \in B\}$ of $B$ in $P$ is a subcomodule of $P$. On the other hand, the formula $p h=h^{[1]} p h^{[2]}$ defines a right action on $Z_{B}(P)$ called the Miyashita-Ulbrich action. This action and coaction satisfy the Yetter-Drinfeld compatibility condition [10, (3.11)]. The following proposition modifies the Miyashita-Ulbrich action so as to obtain stable anti-Yetter-Drinfeld modules.

Proposition 1.5 Let $B \subseteq P$ be a Hopf-Galois $H$-extension such that $B$ is central in $P$. Then $P$ is a right-right stable anti-Yetter-Drinfeld module via the action $p h=\left(S^{-1}(h)\right)^{[2]} p\left(S^{-1}(h)\right)^{[1]}$ and the right coaction on $P$.

The simplest examples are obtained for $P=H$. A broader class is given by the so-called Galois objects [2]. Then quantum-group coverings at roots of unity provide examples with central coinvariants bigger than the ground field (see [9] and examples therein).

\section{Cyclic cohomology of module algebras and coalgebras}

An algebra $A$ which is a module over a Hopf algebra $H$ such that $h(a b)=\left(h^{(1)} a\right)\left(h^{(2)} b\right)$ and $h 1=\varepsilon(h)$, for all $h \in H, a, b \in A$, is called an $H$-module algebra. Similarly, a coalgebra $C$ which is a module over a Hopf algebra $H$ such that $\Delta(h c)=\left(h^{(1)} c^{(1)}\right) \otimes\left(h^{(2)} c^{(2)}\right)$ and $\varepsilon(h c)=\varepsilon(h) \varepsilon(c)$, for all $h \in H$ and $c \in C$, is called an $H$-module coalgebra. In this section we construct cyclic modules for both module algebras and coalgebras.

We begin with the coalgebra case. First, we take a left $H$-comodule $M, H$-module coalgebra $C$, and define an auxiliary simplicial complex $\mathcal{C}^{n}(C, M):=M \otimes C^{\otimes(n+1)}=(M \otimes C) \otimes C^{\otimes n}, n \in \mathbb{N}$, by endowing $M \otimes C$ with the bicomodule structure $M_{\otimes C} \Delta(m \otimes c)=m^{(-1)} c^{(1)} \otimes\left(m^{(0)} \otimes c^{(2)}\right)$, $\Delta_{M \otimes C}(m \otimes c)=\left(m \otimes c^{(1)}\right) \otimes c^{(2)}$. Then the standard formulas for faces and degeneracies on the complex $\left\{\text { bicomodule } \otimes C^{\otimes n}\right\}_{n \in \mathbb{N}}$ translate immediately into

$$
\begin{aligned}
\delta_{i}\left(m \otimes c_{0} \otimes \cdots \otimes c_{n-1}\right) & =m \otimes c_{0} \otimes \cdots \otimes c_{i}^{(1)} \otimes c_{i}^{(2)} \otimes c_{n-1}, \quad 0 \leq i<n \quad \text { (faces), } \\
\delta_{n}\left(m \otimes c_{0} \otimes \cdots \otimes c_{n-1}\right) & =m^{(0)} \otimes c_{0}^{(2)} \otimes c_{1} \otimes \cdots \otimes c_{n-1} \otimes m^{(-1)} c_{0}^{(1)} \quad \text { (flip-over face), } \\
\sigma_{i}\left(m \otimes c_{0} \otimes \cdots \otimes c_{n+1}\right) & =m \otimes c_{0} \otimes \cdots \otimes \varepsilon\left(c_{i+1}\right) \otimes \cdots \otimes c_{n+1}, \quad 0 \leq i \leq n \quad \text { (degeneracies). }
\end{aligned}
$$


It is straightforward to check that these morphisms together with

$$
\tau_{n}\left(m \otimes c_{0} \otimes \cdots \otimes c_{n}\right)=m^{(0)} \otimes c_{1} \otimes \cdots \otimes c_{n} \otimes m^{(-1)} c_{0}
$$

form a paracyclic structure on $\left\{\mathcal{C}^{n}(C, M)\right\}_{n \in \mathbb{N}}$. Now let us assume that $M$ is also a right $H$ module. We can treat $C^{\otimes(n+1)}$ as a left $H$-module via the diagonal action (i.e., $h\left(c_{0} \otimes \cdots \otimes c_{n}\right)=$ $\left.h^{(1)} c_{0} \otimes \cdots \otimes h^{(n+1)} c_{n}\right)$ and define the quotient (invariant) complex $\mathcal{C}_{H}^{n}(C, M):=M \otimes_{H} C^{\otimes(n+1)}$, $n \in \mathbb{N}$. Except for $\tau_{n}$ and $\delta_{n}$ it is clear that the aforementioned morphisms are well defined on the quotient complex. The key result of this paper is that $\tau_{n}$ is well defined if and only if $M$ is an anti-Yetter-Drinfeld module. More precisely, we have:

Theorem 2.1 Let $M$ be a left $H$-comodule and a right $H$-module. Then the map $\tau_{n}$ given by the formula (2.4) is well defined on $M \otimes_{H} C^{\otimes(n+1)}$ for any $n \in \mathbb{N}$ and any $H$-module coalgebra $C$ if and only if $M$ is an anti-Yetter-Drinfeld module. If furthermore $M$ is stable, then $\left\{\mathcal{C}_{H}^{n}(C, M)\right\}_{n \in \mathbb{N}}$ is a cyclic module via (2.1)-(2.4).

Proof. Note first that $\tau_{n}$ is well defined on $M \otimes_{H} C^{\otimes(n+1)}$ if and only if

$$
m^{(0)} \otimes_{H}\left(h^{(2)}\left(c_{1} \otimes \ldots \otimes c_{n}\right) \otimes m^{(-1)} h^{(1)} c_{0}\right)=(h m)^{(0)} \otimes_{H}\left(c_{1} \otimes \ldots \otimes c_{n} \otimes(h m)^{(-1)} c_{0}\right) .
$$

The equality $m^{(0)} \otimes_{H}\left(h^{(2)} \otimes m^{(-1)} h^{(1)}\right)=(h m)^{(0)} \otimes_{H}\left(1 \otimes(h m)^{(-1)}\right)$ evidently implies (2.5) for any $n$ and $C$, and follows if we assume that (2.5) holds for any $n$ and $C$. (E.g., take $n=2, C=H$ and $c_{0}=1=c_{1}$.) Now, observe that there is an isomorphism $\Phi: H \otimes H \rightarrow H \otimes H, \Phi(h \otimes k)=$ $h^{(1)} \otimes S\left(h^{(2)}\right) k, \Phi^{-1}(h \otimes k)=h^{(1)} \otimes h^{(2)} k$. $\Phi$ is left $H$-linear if we view the domain as a left $H$-module via the diagonal action and the counter-domain as a left $H$-module via the multiplication in the first factor. Thus we have an isomorphism $M \otimes_{H}(H \otimes H) \stackrel{\text { id } \otimes_{H} \Phi}{\longrightarrow}\left(M \otimes_{H} H\right) \otimes H \cong M \otimes H$. Applying this isomorphism to the equality below the equation (2.5) yields $m^{(0)} h^{(2)} \otimes S\left(h^{(3)}\right) m^{(-1)} h^{(1)}=$ $(h m)^{(0)} \otimes(h m)^{(-1)}$. This is equivalent to the anti-Yetter-Drinfeld condition, as desired. Next, since $\delta_{n}=\tau_{n} \delta_{0}$, all morphisms are well defined on $\mathcal{C}_{H}^{n}(C, M)$, if $M$ is an anti-Yetter-Drinfeld module. If $M$ is also stable, then the equality $\left(\tau_{n}\right)^{n+1}\left(m \otimes_{H}\left(c_{0} \otimes \cdots \otimes c_{n}\right)\right)=m^{(0)} m^{(-1)} \otimes_{H}\left(c_{0} \otimes \cdots \otimes c_{n}\right)$ entails that $\left(\tau_{n}\right)^{n+1}=\mathrm{id}$, as needed.

For $C=H$ and $M={ }^{\sigma} k_{\delta}$, the complex $\left\{\mathcal{C}_{H}^{n}(C, M)\right\}_{n \in \mathbb{N}}$ becomes the cyclic module of ConnesMoscovici [7]. As an intermediate step, one can take the Hopf cotriples of [11].

We can proceed much the same way in the algebra case. Again, we first have an auxiliary complex $\mathcal{C}^{n}(A, M):=\operatorname{Hom}\left(M \otimes A^{\otimes(n+1)}, k\right) \cong \operatorname{Hom}\left(A^{\otimes n}, \operatorname{Hom}(M \otimes A, k)\right)$. Here $A$ is a left $H$-module algebra and $M$ a left $H$-comodule. The formulas $b(m \otimes a)=m^{(0)} \otimes\left(S^{-1}\left(m^{(-1)}\right) b\right) a$, $(m \otimes a) b=m \otimes a b$ turn $M \otimes A$, and consequently $\operatorname{Hom}(M \otimes A, k)$, into a bimodule. Thus we can use the standard formulas $\left[14\right.$, p.37] to define a simplicial structure on $\left\{\mathcal{C}^{n}(A, M)\right\}_{n \in \mathbb{N}}$. To define an invariant subcomplex, we assume now $M$ to be also a right $H$-module, view $A^{\otimes(n+1)}$ as a left $H$-module via the diagonal action, $M \otimes A^{\otimes(n+1)}$ as a right $H$-module via $(m \otimes \widetilde{a}) h=$ $m h^{(1)} \otimes S\left(h^{(2)}\right) \widetilde{a}$, and $k$ as a right $H$-module via the counit map. Then we can put $\mathcal{C}_{H}^{n}(A, M):=$ $\operatorname{Hom}_{H}\left(M \otimes A^{\otimes(n+1)}, k\right), n \in \mathbb{N}$. Also along the same lines, one can prove that if $M$ satisfies the anti-Yetter-Drinfeld condition, then the morphisms

$$
\begin{aligned}
& \left(\delta_{i} f\right)\left(m \otimes a_{0} \otimes \ldots \otimes a_{n}\right)=f\left(m \otimes a_{0} \otimes \ldots \otimes a_{i} a_{i+1} \otimes \ldots \otimes a_{n}\right), \quad 0 \leq i<n, \\
& \left(\delta_{n} f\right)\left(m \otimes a_{0} \otimes \ldots \otimes a_{n}\right)=f\left(m^{(0)} \otimes\left(S^{-1}\left(m^{(-1)}\right) a_{n}\right) a_{0} \otimes a_{1} \otimes \ldots \otimes a_{n-1}\right), \\
& \left(\sigma_{i} f\right)\left(m \otimes a_{0} \otimes \ldots \otimes a_{n}\right)=f\left(m \otimes a_{0} \otimes \ldots \otimes a_{i} \otimes 1 \otimes \ldots \otimes a_{n}\right), \quad 0 \leq i \leq n, \\
& \left(\tau_{n} f\right)\left(m \otimes a_{0} \otimes \ldots \otimes a_{n}\right)=f\left(m^{(0)} \otimes\left(S^{-1}\left(m^{(-1)}\right) a_{n}\right) \otimes a_{0} \otimes \ldots \otimes a_{n-1}\right),
\end{aligned}
$$

define a paracyclic structure on $\left\{\mathcal{C}_{H}^{n}(A, M)\right\}_{n \in \mathbb{N}}$. Adding the stability assumption on $M$, one obtains: 
Theorem 2.2 If $M$ is a stable anti-Yetter-Drinfeld module and $A$ is a left $H$-module algebra, then the maps (2.6)-(2.9) define a cyclic module structure on $\left\{\mathcal{C}_{H}^{n}(A, M)\right\}_{n \in \mathbb{N}}$.

For $H=k=M$ we recover the usual cyclic cohomology of algebras. For the Laurent polynomial Hopf algebra $\left(H=k\left[\sigma, \sigma^{-1}\right]\right)$ and $M={ }^{\sigma} k_{\varepsilon}$ we obtain the twisted cyclic cohomology [13]. We can also take as a stable anti-Yetter-Drinfeld module a Hopf algebra $K$ thought of as a left comodule over itself via the coproduct, and as a right module over itself via $k \cdot h=S\left(h^{(2)}\right) k h^{(1)}$. Then we arrive at the construction considered in [1]. This is a special case of the construction in Proposition 1.5: $P=K^{c o p}=H$.

Finally, if we take $M={ }^{\sigma} k_{\delta}$, we can view a $\delta$-invariant $\sigma$-trace of [7, Definition 1] as a closed 0 -cocycle in our complex. On the other hand, if $\mathbb{Q} \subseteq k$, we can define cyclic cohomology as follows: $\mathrm{b}_{n}:=\sum_{i=0}^{n}(-1)^{i} \delta_{i}, \mathcal{Z}_{H}^{n}(*, M):=\left.\operatorname{Ker} \mathrm{b}_{n}\right|_{\operatorname{Ker}\left(\tau_{n}-(-1)^{n}\right)}, \mathcal{B}_{H}^{n}(*, M):=\left.\operatorname{Imb} \mathrm{b}_{n-1}\right|_{\operatorname{Ker}\left(\tau_{n-1}-(-1)^{n-1}\right)}$, $H C_{H}^{n}(*, M):=\mathcal{Z}_{H}^{n}(*, M) / \mathcal{B}_{H}^{n}(*, M), n \in \mathbb{N}$. This brings us to the following generalization of [7, Proposition 1]:

Proposition 2.3 Let $C$ be a left $H$-module coalgebra, $A$ a left $H$-module algebra, and $M$ a stable right-left anti-Yetter-Drinfeld over $H$. Assume also that $\mathbb{Q} \subseteq k$ and $C$ acts on $A$ in the sense that we have a linear map $C \otimes A \rightarrow A$ satisfying $c(a b)=\left(c^{(1)} a\right)\left(c^{(2)} b\right), c 1=\varepsilon(c), h(c a)=(h c) a$, for all $h \in H, c \in C, a, b \in A$. Then, for all $n \in \mathbb{N}$, the formulas

$$
\begin{gathered}
\left(\left[m \otimes_{H}\left(c_{0} \otimes \ldots \otimes c_{n}\right)\right] \# f\right)\left(a_{0} \otimes \ldots \otimes a_{n}\right)=f\left(m \otimes_{H}\left(c_{0} a_{0}\right) \ldots\left(c_{n} a_{n}\right)\right) \\
\left.\left(\left(m \otimes_{H} c\right) \#[f]\right)\left(a_{0} \otimes \ldots \otimes a_{n}\right)=f\left(m \otimes_{H}\left(c^{(1)} a_{0}\right) \otimes \ldots \otimes c^{(n+1)} a_{n}\right)\right)
\end{gathered}
$$

define, respectively, the following homomorphisms:

$$
H C_{H}^{n}(C, M) \otimes \mathcal{Z}_{H}^{0}(A, M) \stackrel{\#_{n, 0}}{\longrightarrow} H C^{n}(A), \quad \mathcal{Z}_{H}^{0}(C, M) \otimes H C_{H}^{n}(A, M) \stackrel{\#_{0, n}}{\longrightarrow} H C^{n}(A) .
$$

We conjecture that one can construct along these line a cup product between the Hopf-cyclic cohomology of a module coalgebra and the Hopf-cyclic cohomology of a module algebra. This would generalize in some sense the cup product in [5, p.105].

\section{Cyclic theory for comodule algebras}

In this section we define cyclic cohomology with coefficients in a right-right stable anti-YetterDrinfeld module, and cyclic homology with coefficients in a left-left stable anti-Yetter-Drinfeld module. The latter case generalizes theory introduced [11]. In both cases, we assume that $A$ is a right $H$-comodule algebra. Our strategy for constructing cyclic theory is much as before: bimodule structure $\leadsto$ simplicial structure $\leadsto$ paracyclic structure $\leadsto$ invariant complex $\leadsto$ cyclic theory. In the cohomology case, we define the invariant subcomplex as $\mathcal{C}^{n, H}(A, M):=\operatorname{Hom}^{H}\left(A^{\otimes(n+1)}, M\right)$, $n \in \mathbb{N}$. Here $M$ is a right-right stable anti-Yetter-Drinfeld module, and $A^{\otimes(n+1)}$ is viewed as a right comodule via the diagonal coaction. The following maps define, respectively, faces, degeneracies and cyclic operators on $\left\{\mathcal{C}^{n, H}(A, M)\right\}_{n \in \mathbb{N}}$.

$$
\begin{aligned}
& \left(\delta_{i} f\right)\left(a_{0} \otimes \ldots \otimes a_{n}\right)=f\left(a_{0} \otimes \ldots \otimes a_{i} a_{i+1} \otimes \ldots \otimes a_{n}\right), \quad 0 \leq i<n, \\
& \left(\delta_{n} f\right)\left(a_{0} \otimes \ldots \otimes a_{n}\right)=f\left(a_{n}^{(0)} a_{0} \otimes a_{1} \otimes \ldots \otimes a_{n-1}\right) a_{n}^{(1)}, \\
& \left(\sigma_{i} f\right)\left(a_{0} \otimes \ldots \otimes a_{n}\right)=f\left(a_{0} \otimes \ldots \otimes a_{i} \otimes 1 \otimes \ldots \otimes a_{n}\right), \quad 0 \leq i<n, \\
& \left(\tau_{n} f\right)\left(a_{0} \otimes \ldots \otimes a_{n}\right)=f\left(a_{n}^{(0)} \otimes a_{0} \otimes \ldots \otimes a_{n-1}\right) a_{n}^{(1)} .
\end{aligned}
$$


In the homology case, we take a left-left stable anti-Yetter-Drinfeld module $M$ and define the invariant subcomplex via the cotensor product: $\mathcal{C}_{n}^{H}(A, M):=A^{\otimes(n+1)} \square_{H} M, n \in \mathbb{N}$. (Recall that $X \square_{H} Y:=\operatorname{Ker}\left(\Delta_{X} \otimes\right.$ id - id $\left.\otimes_{Y} \Delta\right)$.) Here, again, we view $A^{\otimes(n+1)}$ as a right $H$-comodule via the diagonal coaction. The following homomorphisms establish, respectively, the desired faces, degeneracies and cyclic operators on $\left\{\mathcal{C}_{n}^{H}(A, M)\right\}_{n \in \mathbb{N}}$.

$$
\begin{aligned}
& d_{i}\left(a_{0} \otimes \ldots \otimes a_{n} \otimes m\right)=a_{0} \otimes \ldots \otimes a_{i} a_{i+1} \otimes \ldots \otimes a_{n} \otimes m, \quad 0 \leq i<n, \\
& d_{n}\left(a_{0} \otimes \ldots \otimes a_{n} \otimes m\right)=a_{n}^{(0)} a_{0} \otimes a_{1} \ldots \otimes a_{n-1} \otimes a_{n}^{(1)} m, \\
& s_{i}\left(a_{0} \otimes \ldots \otimes a_{n} \otimes m\right)=a_{0} \otimes \ldots \otimes a_{i} \otimes 1 \otimes \ldots \otimes a_{n} \otimes m, \quad 0 \leq i \leq n, \\
& t_{n}\left(a_{0} \otimes \ldots \otimes a_{n} \otimes m\right)=a_{n}^{(0)} \otimes a_{0} \otimes \ldots \otimes a_{n-1} \otimes a_{n}^{(1)} m .
\end{aligned}
$$

Theorem 3.1 Let $A$ be a right $H$-comodule algebra and $M$ a right-right (resp. left-left) stable anti-Yetter-Drinfeld module over $H$. Then the formulas (3.1)-(3.4) (resp. (3.5)-(3.8)) define a cyclic module structure on $\left\{\operatorname{Hom}^{H}\left(A^{\otimes(n+1)}, M\right)\right\}_{n \in \mathbb{N}}\left(\right.$ resp. $\left.\left\{A^{\otimes(n+1)} \square_{H} M\right\}_{n \in \mathbb{N}}\right)$.

Now one can either define the cyclic cohomology (resp. homology) of $A$ with coefficients in $M$ as the total cohomology (resp. homology) of an appropriate double complex [14, p.77 and p.72], or assume that $\mathbb{Q} \subseteq k$ and proceed as above Proposition 2.3.

Acknowledgements. This work was partially supported by the Marie Curie Fellowship HPMF-CT-200000523 and KBN grant 2 P03A 01324 (P.M.H.). P.M.H. is also grateful to the University of Western Ontario for its hospitality and financial support, and to the participants of his Hopf-cyclic cohomology workshop for their interest and encouragement. Special thanks are due to M. Furuta and P. Schauenburg for their helpful remarks, and to T. Brzeziński for his help with finding references. All authors are grateful to R. Taillefer for the French translation.

\section{References}

[1] Akbarpour, A.; Khalkhali, M.: Equivariant cyclic cohomology of Hopf module algebras. J. reine angew. Math. 559 (2003) 137-152.

[2] Caenepeel, S.: Brauer groups, Hopf algebras and Galois theory. K-Monographs in Mathematics, 4 . Kluwer Academic Publishers, Dordrecht, 1998.

[3] Caenepeel, S.; Militaru, G.; Zhu, S.: Frobenius and separable functors for generalized module categories and nonlinear equations. Lecture Notes in Mathematics, 1787, Springer-Verlag, Berlin, 2002.

[4] Chevalley, C.; Eilenberg, S.: Cohomology theory of Lie groups and Lie algebras. Trans. Amer. Math. Soc. 63 (1948) 85-124.

[5] Connes, A.: Noncommutative differential geometry. Inst. Hautes Ètudes Sci. Publ. Math. 62 (1985) $257-360$.

[6] Connes, A.; Moscovici, H.: Hopf algebras, cyclic cohomology and the transverse index theorem. Commun. Math. Phys. 198 (1998) 199-246.

[7] Connes, A.; Moscovici, H.: Cyclic cohomology and Hopf algebras. Lett. Math. Phys. 48 (1999) 97-108.

[8] Connes, A.; Moscovici, H.: Cyclic cohomology and Hopf algebra symmetry. Lett. Math. Phys. 52 (2000) 1-28.

[9] Dąbrowski, L.; Hajac, P.M.; Siniscalco, P.: Explicit Hopf-Galois description of $S L_{e^{2 i \pi / 3}}(2)$-induced Frobenius homomorphisms. Enlarged proceedings of the ISI GUCCIA workshop on quantum groups, non commutative geometry and fundamental physical interactions, D. Kastler, M. Rosso, T. Schucker (eds.), Commack - New York, Nova Science Pub, Inc., pp.279-298, 1999.

[10] Doi, Y.; Takeuchi, M.: Hopf-Galois extensions of algebras, the Miyashita-Ulbrich action, and Azumaya algebras. J. Algebra 121 (1989) 488-516.

[11] Khalkhali, M.; Rangipour, B.: Invariant cyclic homology. To appear in $K$-Theory, math/0207118.

[12] Khalkhali, M.; Rangipour, B.: A new cyclic module for Hopf algebras. K-Theory 27 (2002) 111-131.

[13] Kustermans, J.; Murphy, G.J.; Tuset, L.: Differential calculi over quantum groups and twisted cyclic cocycles. J. Geom. Phys. 44 (2003) 570-594.

[14] Loday, J.-L.: Cyclic Homology. Springer-Verlag, 1998. 